\title{
Repeated changes of the molecular subtype in gastric metastasis from breast cancer: A case report
}

\author{
JUN NAKAMURA ${ }^{1}$, KEIICHIRO OKUYAMA ${ }^{1}$, HIROFUMI SATO ${ }^{1}$, \\ YUKIE YODA $^{1}$, KEITA KAI $^{2}$ and HIROKAZU NOSHIRO ${ }^{1}$ \\ ${ }^{1}$ Department of Surgery, Saga University Faculty of Medicine; \\ ${ }^{2}$ Department of Pathology, Saga University Hospital, Saga 849-8501, Japan
}

Received September 2, 2015; Accepted December 18, 2015

DOI: $10.3892 / \operatorname{mco} .2016 .795$

\begin{abstract}
Gastric metastasis from breast cancer is clinically diagnosed rarely. The present study described an interesting and valuable case of gastric metastasis from breast cancer, which showed repeated changes of the molecular subtype with an impact on the choice of treatment. A 42-year-old woman underwent mastectomy with axillary lymph node dissection for an invasive lobular carcinoma of the left breast. The patient received gastroscopy due to an epigastric pain during the adjuvant chemotherapy. The endoscopic examination revealed an erosive lesion at the posterior wall of the gastric body. The gastric lesion was immunohistochemically diagnosed as a metastatic disease from the breast cancer. The patient initially received hormone therapy, according to the subtype of the primary and the metastatic diseases. The gastric lesion initially disappeared; however, a relapsed lesion transformed into luminal human epidermal growth factor receptor 2 type from luminal type. Subsequently, the metastatic lesions underwent repeated subtype changing, which created difficultly when deciding the treatment strategy. The molecular profile of breast cancer can change during the treatment, resulting in the treatment resistance observed in certain cases. Therefore, the optimal treatment must be selected, according to the changed subtype.
\end{abstract}

\section{Introduction}

Breast cancer (BC) is the most frequently diagnosed type of cancer and the leading cause of cancer-associated mortality in women worldwide (1). The molecular profiling of $\mathrm{BC}$, based on gene expression, is useful to characterize this heterogeneous disease. The molecular subtypes of $\mathrm{BC}$

Correspondence to: Dr Jun Nakamura, Department of Surgery, Saga University Faculty of Medicine, 5-1-1 Nabeshima, Saga 849-8501, Japan

E-mail: jun19760712@hotmail.com

Key words: breast cancer, gastric metastasis, molecular subtype, subtype change, heterogeneity provide important predictive and prognostic information. BC can be classified into human epidermal growth factor receptor (HER)2-positive/enriched, hormone-receptor (estrogen and/or progesterone) positive (luminal), positive for both (luminal HER2) or triple negative BC (negative for estrogen and progesteron receptors, and HER2) (2). The treatment strategy for metastatic BC is designed according to a several factors, including the performance status, site of metastasis and the molecular subtype of the disease (3). The present report described a case of gastric metastasis from $\mathrm{BC}$, which showed repeated changes of the molecular subtype, making it difficult to select the appropriate treatments.

\section{Case report}

A 42-year-old woman underwent a mastectomy with axillary lymph node dissection for the left BC in August 2006. The primary tumor was pathologically diagnosed as invasive lobular carcinoma (Fig. 1A and B), $4.5 \mathrm{~cm}$ in diameter, and lymph node metastasis was observed in $37 / 51$ harvested nodes. The disease was stage IIIC, according to the UICC Tumor Node Metastasis (TNM) classification for BC (T3 N3 M0) (4). Immunohistochemical examinations revealed that the hormone receptors were positive, while HER2 status was negative (Fig. 1C-E). At 2 months following the operation, the patient underwent an endoscopic examination for a complaint of an epigastric pain, which occurred during the postoperative adjuvant chemotherapy. The gastroscopy revealed an erosive lesion at the posterior wall of the gastric body (Fig. 2). A biopsy specimen obtained from the gastric lesion contained lobular carcinoma, which was morphologically similar to signet ring cell carcinoma, as frequently exhibited in primary gastric cancer. The immunohistochemical examinations (Fig. 3A) revealed positive staining for hormone receptors, mammaglobin and gross cystic disease fluid protein (GCDFP)-15, which was similar to the findings observed in the primary $\mathrm{BC}$ lesion. These findings suggested that the gastric lesion was a metastatic disease from the $\mathrm{BC}$.

The patient received hormone therapy using an aromatase inhibitor following the completion of the adjuvant chemotherapy. About 2 years later, in July 2008, the gastric lesion disappeared successfully by the treatments described above. However, the patient had to restart chemotherapy again in 

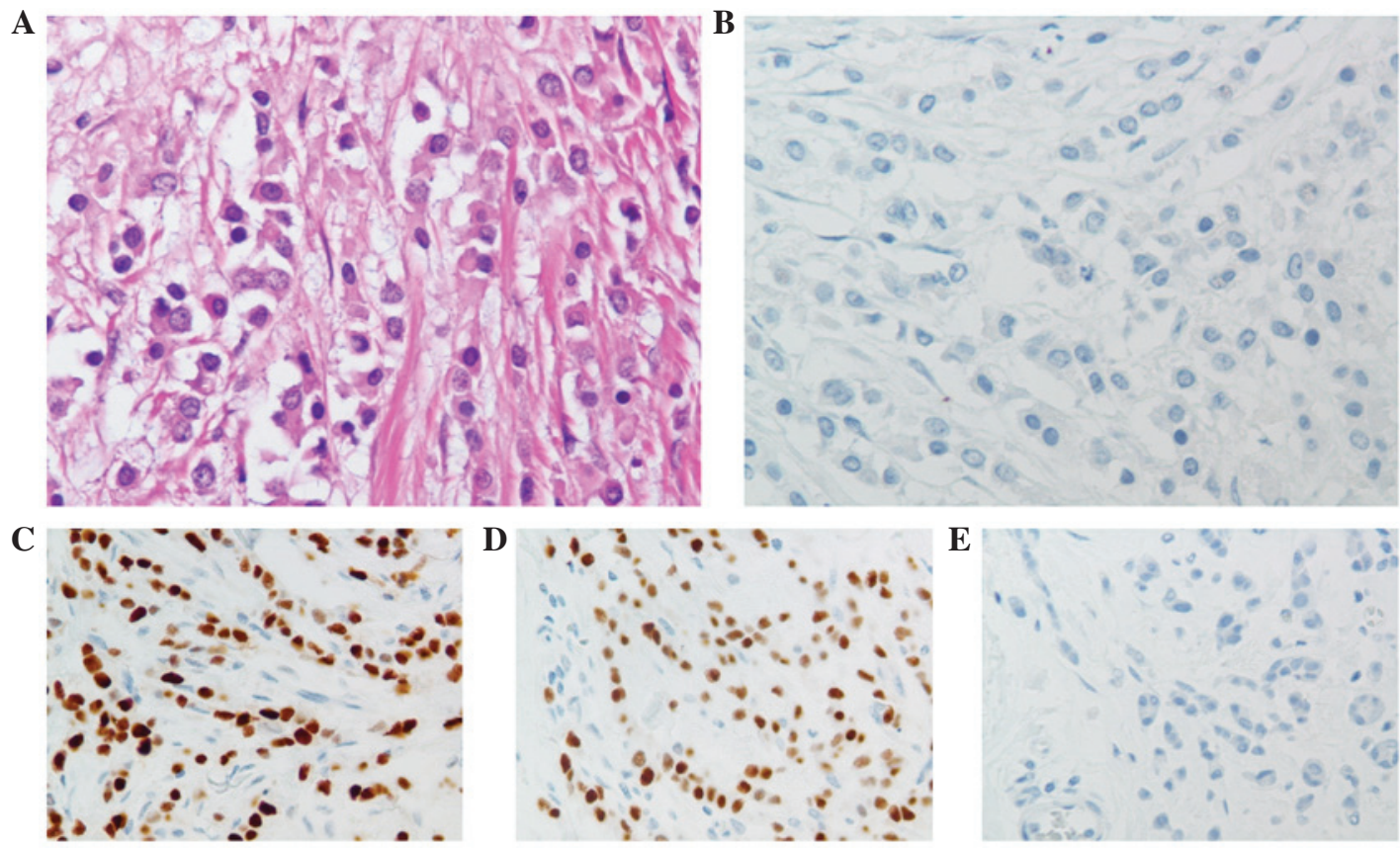

Figure 1. Pathological findings of the primary breast cancer. (A) The microscopic appearance of the tumor indicated invasive lobular carcinoma exhibiting poorly-adhesive cancer cells, as determined by Heamtoxylin and eosin staining with (B) negative staining for E-cadherin. Positive hormone-receptor status of (C) estrogen receptor and (D) progesterone receptor, and (E) negative HER2 status suggested the subtype of the tumor is luminal type. (magnification, x400; HER2 score $=0$, according to the American Society of Clinical Oncology/College of American Pathologists guidelines for HER2 testing. HER, human epidermal growth factor receptor.

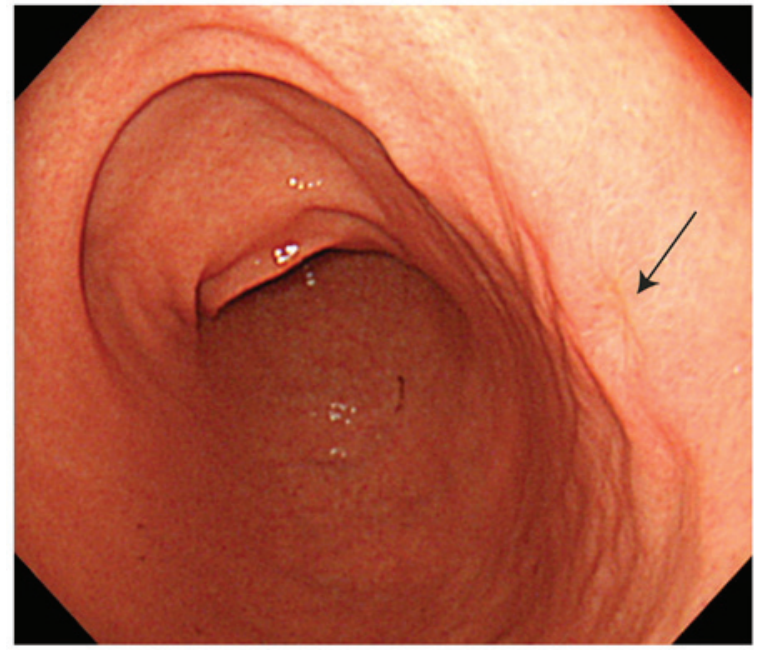

Figure 2. Gastroscopy revealed an erosive lesion (arrow) at the posterior wall of the middle third of the gastric body.

March 2009 due to a relapse of the disease at the fundus, angle and antrum beside the previous body lesion on the stomach. In addition, the molecular subtype of the relapsed lesions had changed to the luminal HER2 type (Fig. 3B) from the luminal type; therefore, chemotherapy, including trastuzumab, was selected. Subsequently, in July 2012, as the molecular subtype of the disease changed again to the triple negative type (Fig. 3C), the patient was administered chemotherapy alone. Although she was treated with chemotherapy until May 2014, the accumulating side effects of the treatment made it difficult for her to receive any additional chemotherapy. Surgical resection of the stomach was considered as a therapeutic option since the recurrent disease had only been observed only in the stomach for $\sim 8$ years. Laparoscopic total gastrectomy was planned in July 2014; however, only an exploratory laparoscopy was performed due to the disseminated disease on peritoneum in the entire abdomen, which was not diagnosed preoperatively. Chemotherapy was restarted following the operation and the molecular subtype of the disease had changed into luminal type (Fig. 3D) from the triple negative type in December 2014.

A total of 8.5 years have passed since the mastectomy and the patient is still receiving chemotherapy for the gastric metastases and the peritoneal dissemination without occurrence of any other novel lesion.

\section{Discussion}

The rate of gastrointestinal (GI) metastasis from $\mathrm{BC}$ is considered to be $<1 \%$ in the clinical setting $(5,6)$, which is markedly lower compared with the 8-35\% observed in the autopsy series $(7,8)$. Therefore, GI metastasis from BC may often be overlooked due to the lack of serious symptoms. Furthermore, Borst and Ingold (6) demonstrated that the rate of GI metastasis was significantly higher in patients with invasive lobular carcinoma compared with invasive ductal carcinoma ( 4.5 vs. $0.5 \%$ ). Similar to the present case, if the gastric lesion appears to be lobular carcinoma, determining the primary site of disease is required for further treatment planning. In such a case, immunohistochemical staining for mammaglobin and GCDFP-15 are useful to distinguish between primary gastric cancer and the metastatic disease from the BC $(9,10)$. Estrogen receptor (ER) and progesterone receptor expression are observed in 32 and $12 \%$ of primary gastric cancers, respectively, indicating that their specificity is low (11-13). 


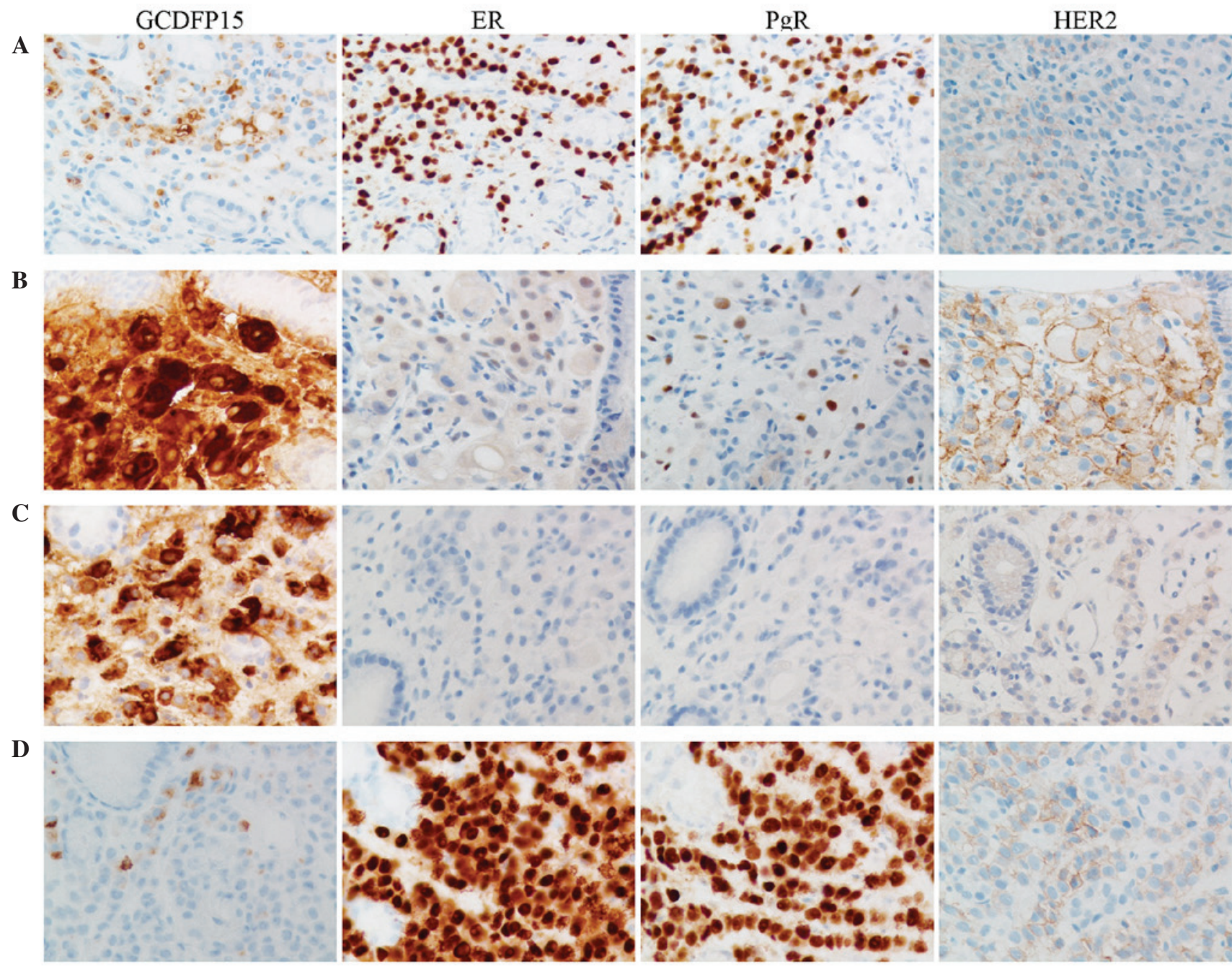

Figure 3. IHC of the biopsy specimens obtained from the gastric lesions. (A) IHC series in October 2006. Focally positive for GCDFP15 staining was observed. ER and PgR were markedly expressed and HER2 staining status was negative (HER2 score, 1+). (B) IHC series in March 2009. Cancer cells exhibited markedly positive staining for GCDFP15. Hormone receptors were focally positive and HER2 staining status was positive (HER2 score, 3+). (C) IHC series in July 2012. Cancer cells exhibited marked staining of GCFDP15 and the absence of ER, PgR and HER2 (HER2 score, 1+). (D) IHC series in December 2014 Focally positive for GCDFP15 staining was observed. The staining status of hormone receptors are diffusely positive for both ER and PgR. The staining score of HER2 was 2+. HER2 scores were according to the American Society of Clinical Oncology/College of American Pathologists guidelines for HER2 testing. Magnification, x400. IHC, immunohistochemistry; PgR, progesterone receptor; HER, human epidermal growth factor receptor; ER, estrogen receptor; GCDFP, gross cystic disease fluid protein.

The treatment recommendation for gastric metastases from $\mathrm{BC}$ is typically a systemic treatment based on their molecular subtype; however, surgical intervention should be limited to palliation or for certain cases of solitary metastases (14).

The present case was initially treated with hormone therapy following adjuvant chemotherapy. Both the original molecular subtypes of the primary BC and gastric metastases were the luminal type; therefore, treatment using an aromatase inhibitor was selected. Although the metastatic lesions once disappeared, the relapsed lesions were transformed into the luminal HER2 type. The mechanisms contributing to hormone resistance are hypothesized to include loss or modification of the ER expression, regulation of alternative signal transduction pathways, altered expression of specific microRNAs, changes in the balance of co-regulatory proteins and genetic polymorphisms involved in tamoxifen metabolic activity (15). In the present case, the lesions initially negative for HER 2 expression shifted to positive expression. It was therefore suggested that activation of the HER2 signaling pathway was the predominant mechanism underlying the acquired resistance to hormone therapy. A recent study using a mouse xenograft model also demonstrated that 3/26 (11\%) tumors originally negative for HER 2 became amplified and/or overexpressed upon the acquisition of hormone resistance (16). Furthermore, Yang et al (17) reported that a HER2 status shift was observed in $17 / 113(15.0 \%)$ patients who received neoadjuvant chemotherapy (NAC). Among this series, a gain of HER 2 was observed in $8 / 17$ patients. The molecular subtype of the patient in the present report further changed to the triple negative type from luminal HER2 type during the treatment. The loss of ER expression is another mechanism associated with hormone resistance, as described above. Whereas, the mechanisms that contribute to trastuzumab resistance include obstacles preventing trastuzumab binding to HER2, upregulation of signaling pathways downstream of HER2, signaling through alternate pathways and failure to trigger 
an immune-mediated mechanism to destroy tumor cells (18). Previously, two independent studies reported that the loss of HER2 expression following NAC was observed in 20-27.5\% of patients who initially expressed the HER2 protein $(19,20)$. It was hypothesized that a change in the molecular subtype in the present case may indicate the acquisition of resistance to the treatment via the activation of an alternative receptor pathway, including pathways involving insulin-like growth factor-1R, epidermal growth factor receptor and c-Met. Another explanation of the subtype change may be that the HER2-positive component of BC was suppressed by trastuzumab and the remaining section of the tumor showed progression. In other words, the subtype change reflected the heterogeneity of BC. In any case, clinicians must carefully monitor the molecular behavior of $\mathrm{BC}$ to select the optimal treatment.

In conclusion, it is important to consider the possibility of a subtype change if treatment failure occurs. Repeated biopsies must be performed during the course of the treatment when the primary $\mathrm{BC}$ and/or metastatic lesions are available.

\section{References}

1. Jemal A, Bray F, Center MM, Ferlay J, Ward E and Forman D: Global cancer statistics. CA Cancer J Clin 61: 69-90, 2011.

2. van't Veer LJ, Dai H, van de Vijver MJ, He YD, Hart AA, Mao M, Peterse HL, van der Kooy K, Marton MJ, Witteveen AT, et al: Gene expression profiling predicts clinical outcome of breast cancer. Nature 415: 530-536, 2002.

3. Guarneri V and Conte P: Metastatic breast cancer: Therapeutic options according to molecular subtypes and prior adjuvant therapy. Oncologist 14: 645-656, 2009.

4. Greene FL: Breast tumours. In: TNM classification of malignant tumours. Sobin LH, Gospodarowicz MK and Wittekind C (eds). 7th ed. Wiley-Blackwell, Oxford, pp181-93, 2009.

5. Hoff J, Portet R, Becue J, Fourtanier G and Bugat R: Digestive tract metastases of breast cancers. Ann Chir 37: 281-284, 1983 (In French).

6. Borst MJ and Ingold JA: Metastatic patterns of invasive lobular versus invasive ductal carcinoma of the breast. Surgery 114: 637-641; discussion 641-642, 1993.

7. Washington K and McDonagh D: Secondary tumors of the gastrointestinal tract: Surgical pathologic findings and comparison with autopsy survey. Mod Pathol 8: 427-433, 1995.
8. Caramella E, Bruneton JN, Roux P, Aubanel D and Lecomte P: Metastases of the digestive tract. Report of 77 cases and review of the literature. Eur J Radiol 3: 331-338, 1983.

9. Wang Z, Spaulding B, Sienko A, Liang Y, Li H, Nielsen G, Yub Gong G, Ro JY and Jim Zhai Q: Mammaglobin, a valuable diagnostic marker for metastatic breast carcinoma. Int J Clin Exp Pathol 2: 384-389, 2009.

10. Takeda Y, Tsuta K, Shibuki Y, Hoshino T, Tochigi N, Maeshima AM, Asamura H, Sasajima Y, Ito T and Matsuno Y: Analysis of expression patterns of breast cancer-specific markers (mammaglobin and gross cystic disease fluid protein 15) in lung and pleural tumors. Arch Pathol Lab Med 132: 239-243, 2008.

11. Raju U, Ma CK and Shaw A: Signet ring variant of lobular carcinoma of the breast: A clinicopathologic and immunohistochemical study. Mod Pathol 6: 516-520, 1993.

12. Wick MR, Lillemoe TJ, Copland GT, Swanson PE, Manivel JC and Kiang DT: Gross cystic disease fluid protein-15 as a marker for breast cancer: Immunohistochemical analysis of 690 human neoplasms and comparison with alpha-lactalbumin. Hum Pathol 20: 281-287, 1989.

13. Schwarz RE, Klimstra DS and Turnbull AD: Metastatic breast cancer masquerading as gastrointestinal primary. Am J Gastroenterol 93: 111-114, 1998.

14. Pectasides D, Psyrri A, Pliarchopoulou K, Floros T, Papaxoinis G, Skondra M, Papatsibas G, Macheras A, Athanasas G, Arapantoni-Datioti P and Economopoulos T: Gastric metastases originating from breast cancer: Report of 8 cases and review of the literature. Anticancer Res 29: 4759-4763, 2009.

15. García-Becerra R, Santos N, Díaz L and Camacho J: Mechanisms of resistance to endocrine therapy in breast cancer: Focus on signaling pathways, miRNAs and genetically based resistance. Int J Mol Sci 14: 108-145, 2012.

16. Gutierrez MC, Detre S, Johnston S, Mohsin SK, Shou J, Allred DC, Schiff R, Osborne CK and Dowsett M: Molecular changes in tamoxifen-resistant breast cancer: Relationship between estrogen receptor, HER-2 and p38 mitogen-activated protein kinase. J Clin Oncol 23: 2469-2476, 2005.

17. Yang YF, Liao YY, Li LQ, Xie SR, Xie YF and Peng NF: Changes in ER, PR and HER2 receptors status after neoadjuvant chemotherapy in breast cancer. Pathol Res Pract 209: 797-802, 2013.

18. Pohlmann PR, Mayer IA and Mernaugh R: Resistance to trastuzumab in breast cancer. Clin Cancer Res 15: 7479-7491, 2009.

19. Guarneri V, Dieci MV, Barbieri E, Piacentini F, Omarini C, Ficarra G, Bettelli S and Conte PF: Loss of HER2 positivity and prognosis after neoadjuvant therapy in HER2-positive breast cancer patients. Ann Oncol 24: 2990-2994, 2013.

20. Hirata T, Shimizu C, Yonemori K, Hirakawa A, Kouno T, Tamura K, Ando M, Katsumata N and Fujiwara Y: Change in the hormone receptor status following administration of neoadjuvant chemotherapy and its impact on the long-term outcome in patients with primary breast cancer. Br J Cancer 101: 1529-1536, 2009. 